\title{
MATHEMATICS AND FINANCE
}

This article presents how mathematical tools can be used in finance. It also shows some interconnections among several parts of mathematics as mathematical analysis, numerical analysis and financial mathematics. Linear interpolation is used to calculate unknown interest rate in various parts of financial mathematics.

Key words: mathematics, finance, interest rate, yield to maturity, linear interpolation.

\section{Introduction}

Mathematics is science widely used almost in all areas of human life, so it is possible to claim that it is interdisciplinary science. People intuitively expect mathematics in natural and computer sciences as physics, informatics or in various technical sciences as machine engineering, civil engineering or electrical engineering. The majority of disciplines mentioned above are provided at our university. Consequently mathematics is needed to be taught at each faculty in a suitable way so that aspects of each discipline would be satisfied. Many technical subjects are also somehow connected, we can refer, e.g., to [2] where interconnections between physics and electrical engineering are described.

Mathematics is also present in such spheres of interest as economy and finance. In recent times there have arisen many new problems which needed extension of financial mathematics and mathematical modeling of various economic processes. In this article we focus our attention to using mathematics for solving some problems in finance.

The paper is organized as follows. In section 2 there are briefly described some parts of mathematics of finance as general theory of interest rates, ordinary simple annuities and bond pricing. In section 3 the numerical method of linear interpolation is explained and in the next section it is used to compute unknown interest rates. Section 4 also contains several illustrative examples.

\section{Mathematics of Finance}

Knowledge in the field of mathematics of finance and actuarial science has increased in importance because of the variability and high level of interest rates. Regardless of whether or not your career is in business, understanding how interest is computed on investments and loans is important to you as a consumer.
The study of mathematics of finance usually begins with a simple interest, sometimes used on short-term investments or loans. The simple interest is, by definition, based only on the initial deposit (the principal) which remains fixed during the entire interest period and accumulated value $S$ of principal $P$ at simple interest rate $i$ is calculated according to the formula: $S=P(1+i n)$. Most investments pay a compound interest, i.e., earned interest for each period is added to the principal before the interest is calculated for the next period. The principal grows as the interest is added to it. The account earns an interest on interest in addition to earning interest on the principal. This method is used in longterm investments and a corresponding formula for the accumulated value at compound interest is: $S=P(1+i)^{n}$. The third basic method of calculating the interest is by continuous compounding. It is reasonable to assume that the more frequently the interest is compounded, the larger the compound amount becomes. It is clear that as the number of periods per year increases, the compound amount increases, although not very rapidly. The accumulated value under continuous compounding is: $S=P e^{\delta t}$, where $\delta$ is called force of interest.

For a more detailed explanation we can refer, e. g., to [3], where the theory of financial mathematics is described.

\subsection{General Theory of Interest Rates}

Usually (when using simple, compound or continuous interest) it is supposed that the interest rate is a constant. In reality, when time periods are longer, this is not true. Now, in accordance with [1], let us consider the interest rate to be a function of time, i.e., $i=i(t)$ - which is an effective interest rate at time $t$ : capital of $1 €$ at time $t$ will increase to $1+i(t)$ during 1 time period. The interest rate $i(t)$ is an amount of interest of $1 €$ within time interval $\left[t_{1}, t_{2}\right]$.

\footnotetext{
* Maria Kudelcikova

Department of Mathematics, Faculty of Science, University of Zilina, Slovakia, E-mail: maria.kudelcikova@fpv.uniza.sk
} 
Let $A\left(t_{1}, t_{2}\right)$ be the future value of $1 €$ deposited at time $t_{1}$ and due at time $t_{2}$. The value $A\left(t_{1}, t_{2}\right)$ is called an accumulation factor. For this factor, so called consistence principle is valid, i.e.,

$$
\begin{aligned}
& A\left(t_{0}, t_{n}\right)=A\left(t_{0}, t_{1}\right) \cdot A\left(t_{1}, t_{2}\right) \cdot \ldots \cdot A\left(t_{n-1}, t_{n}\right), \\
& t_{0} \leq t_{1} \leq t_{2} \leq \ldots \leq t_{n} .
\end{aligned}
$$

Let us denote force of interest for time unit by $\delta(t)$. It is defined by formula

$$
\delta(t)=\lim _{h \rightarrow 0^{+}} \frac{A(t, t+h)-1}{h},
$$

supposing that the limit on the right-hand side exists. Let $t_{0} \leq t$, denote $f(t)=A\left(t_{0}, t\right)$. Now, considering that $\delta(t), f(t)$ are continuous functions $t \geq 0$ for and function $f(t)$ is differentiable, we have:
Now we derive the formula for this accumulated value $S$. The equation of value at the end of the term is: $S=R+R(1+i)+$ $+R(1+i)^{2}+\ldots+R(1+i)^{n-1}$.

This is a geometric sequence of $n$ terms with the first term $a_{1}=R$ and ratio $\mathrm{q}=1+i$. Thus, for $S$ we have:

$$
\begin{aligned}
S & =a_{1} \frac{q^{n}-1}{q-1}=R \frac{(1+i)^{n-1}-1}{(1+i)-1}= \\
& =R \frac{(1+i)^{n}-1}{i}=R S_{n \mid i} .
\end{aligned}
$$

Here $s_{n \mid i}$, read "s angle $n$ at $i$," is called the accumulated value of $1 €$ per period, or an accumulation factor for $n$ payments. The factor $s_{n \mid i}$ can be computed directly with a calculator, or found in Tables, listing these factors for certain values of $i$ and $n$.

$$
\begin{gathered}
\delta(t)=\lim _{h \rightarrow 0^{+}} \frac{A(t, t+h)-1}{h}=\lim _{h \rightarrow 0^{+}} \frac{A\left(t_{0}, t\right) \cdot A(t, t+h)-A\left(t_{0}, t\right)}{h \cdot A\left(t_{0}, t\right)}= \\
=\frac{1}{A\left(t_{0}, t\right)} \cdot \lim _{h \rightarrow 0^{+}} \frac{A\left(t_{0}, t\right) \cdot A(t, t+h)-A\left(t_{0}, t\right)}{h}=\frac{1}{f(t)} \lim _{h \rightarrow 0^{+}} \frac{f(t+h)-f(t)}{h}=\frac{1}{f(t)} f^{\prime}(t) .
\end{gathered}
$$

Hence, we obtain the differential equation $f^{\prime}(t)=f(t) \delta(t)$ and its general solution is $f(t)=c e \int_{w}^{\prime \prime}(s) d s$ where $c$ is some suitable constant.

Using the consistence principle, for $A\left(t_{1}, t_{2}\right)$ we have:

$$
A\left(t_{1}, t_{2}\right)=\frac{A\left(t_{0}, t_{2}\right)}{A\left(t_{0}, t_{1}\right)}=\frac{f\left(t_{2}\right)}{f\left(t_{1}\right)}=e^{\int^{\circ} \delta(t) d t} .
$$

If $t_{1}=0, t_{2}=t$ then we obtain $A(0, t) \int_{J_{0}^{\prime}}^{\prime}(s) d s$ and from this

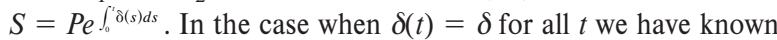
formula for continuous compounding.

In this section it was visible how some nontrivial tools of mathematical analysis (differential equations, integrals) can be utilized in mathematics of finance.

\subsection{Ordinary Simple Annuity}

In this section we derive formulas for calculating accumulated and present values of an ordinary simple annuity, which is a sequence of periodic payments. The accumulated value $\mathrm{S}$ of an ordinary simple annuity of $n$ periodic payments of $R €$ each at an interest rate $i$ per period [see Fig. 1.] is the equivalent dated value of the set of these payments due, at the end of the term of the annuity (which is the date of the last payment).

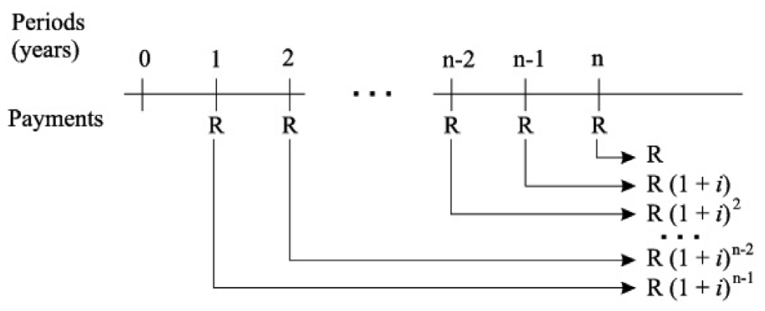

Fig. 1 Ordinary simple annuity
Multiplying both sides of equation $(A V)$ by $(1+i)^{-n}$, we obtain a formula for the present value of ordinary annuity $A$ :

$$
A=R \frac{1-(1+i)^{-n}}{i}=R a_{n \mid i},
$$

where $a_{n \mid i}$ is called a discount factor for $n$ payments and represents the present value of an ordinary simple annuity of $1 €$ per period for $n$ periods, with an interest rate of $i$ per period.

The most efficient way to solve an annuity problem is to make a time diagram, determine the type of annuity, and then apply the proper formula.

In this part it is seen as the geometric sequence theory can be used in financial mathematics.

\subsection{Bonds - introduction}

Financial newspapers list thousands of bonds issued by corporations, municipalities, and the government. In determining which bonds are appropriate for their needs, investors should consider a number of factors, including risk, expected rate of return, and the feasibility of purchasing a short-term or a long-term bond. Tax implications should also be considered.

A bond is a written contract that requires the issuer (borrower) to pay the investor (lender) interest income. Most bonds make fixed coupon payments at a coupon rate $r$ per interest period every year until the bond matures. Bond coupons are like annuity payments. At maturity, the bond issuer must also repay the face value of the bond $F$. The market price of a bond equals its present value $P$. Hence the investor who wishes to realize a rate of return $i$ (until the bond is redeemed or matures) should pay a price equal to the discounted value of the $n$ coupons $C=F r$ plus the discounted value of the face value: 


$$
\begin{aligned}
P & =\frac{C}{(1+i)}+\frac{C}{(1+i)^{2}}+\mathrm{E}+\frac{C+F}{(1+i)^{n}}= \\
& =F r a_{n \mid i}+\frac{F}{(1+i)^{n}},
\end{aligned}
$$

which is equivalent to $P=F+F(r-i) a_{n \mid i}$ known as an alternate purchase price formula.

Since finding the solution for the yield rate per interest period $i$ (often called the yield to maturity) of previous equations is not trivial, the following approximations are used:

1. Hawawini and Vora (1982): $i \approx \frac{C+(F-P) / n}{0.6 P+0.4 F}$.

2. Bond Commercial method (Uhlir and Steiner (1994)):

$$
i \approx \frac{C+(F-P) / n}{P} \text {. }
$$

3. Bond Salesman's method: $i \approx \frac{C+(F-P) / n}{(F+P) / 2}$.

For more accurately results we can use the method of linear interpolation, which is introduced in the next section.

\section{Linear Interpolation}

Linear interpolation is the method of functions approximation when linear functions are used to approximate some functions or various measuring data. Using this method it is also possible to compute arbitrary value $x$ lying in the interval between two known numeric data $x_{1}$ and $x_{1}$ when there are also known values $y_{1}, y_{2}$ and $y$ as it can be seen in Fig. 2.

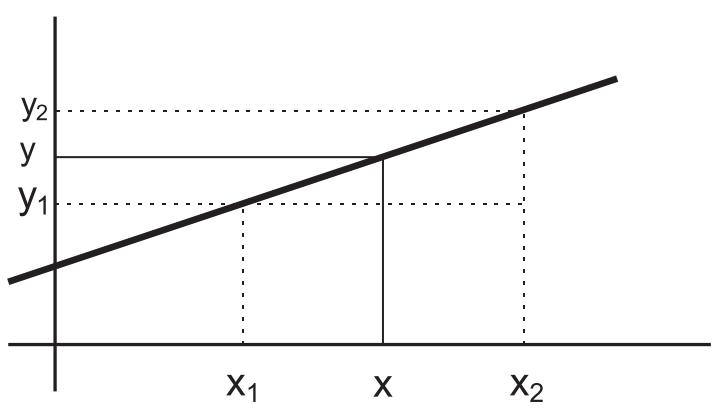

Fig. 2 Sketch for deriving the formula

The easiest way to find a formula for linear interpolation is the triangle similarity theory. We have:

$$
\frac{y_{2}-y_{1}}{x_{2}-x_{1}}=\frac{y-y_{1}}{x-x_{1}} .
$$

From this equation we obtain the following formula for unknown $x$ :

$$
x=x_{1}+\left(x_{2}-x_{1}\right) \frac{y-y_{1}}{y_{2}-y_{1}},
$$

which will be used later for calculating the unknown interest rate and unknown yield to maturity.

\section{Using Linear Interpolation in Mathematics of Finance}

In this section it is showed how linear interpolation can be used in financial mathematics to compute unknown interest rate concerning ordinary annuities and bonds. Some illustrative examples are given, too.

\subsection{Ordinary Simple Annuity}

Using formulae $(A V)$ or $(P V)$ it is possible to compute the other unknown parameters, but it can be seen that the calculating of the unknown interest rate $i$ is not so elementary. Hence when $R, n$ and either $S$ or $A$ are given, the unknown rate $i$, may be determined approximately by linear interpolation. For most practical purposes, linear interpolation gives sufficient accuracy.

F or fixed $n, s_{n \mid i}$ increases when $i$ increases, whereas $a_{n \mid i}$ decreases. In general, the closer the bounds on the interest rate $i$, the better the approximation of $i$ furnished by linear interpolation. We adopt the practice of interpolating between two nominal rates $i_{1}$ and $i_{2}$ that are $1 \%$ apart and using factors $s_{n \mid i}$ and $a_{n \mid i}$ rounded off to 4 decimal places. For the linear interpolation we use formula $(L I)$, where $y_{1}=s_{n \mid i_{1}}, y_{2}=s_{n \mid i_{2}}$ and $y=s_{n \mid i}$ or, respectively $y_{1}=$ $=a_{n \mid i_{1}}, y_{2}=a_{n \mid i_{2}}$, and $y=a_{n \mid i}$.

To obtain a starting value $i_{s}$ to solve an equation $s_{n \mid i}=k$ for unknown $i$ by linear interpolation, we may use the formula from [4]: $i_{s}=\frac{(k / n)^{2}-1}{k}$ and to obtain a starting value to solve an equation $a_{n \mid i}=k$ by linear interpolation, we may use the formula from [4]: $i_{s}=\frac{1-(k / n)^{2}}{k}$.

Now we are in position to give some simple illustrative examples:

\section{Example 1.}

Find the interest rate $i$ at which annual deposits $250 €$ will accumulate to $5000 €$ in 12 years.

We have $R=250, S=5000, n=12, i=$ ? We use the formula $(A V)$, so in our case we have: $250 s_{3 \mid i}=5000$, that means $s_{12 \mid i}=20=k$. To determine the rate $i$, we find two factors $s_{12 \mid i_{1}}$ and $s_{12 \mid i_{2}}$, one greater than 20 and one less than 20, and corresponding values $i_{1}$ and $i_{2}$ that differ by $1 \%$. These values provide upper and lower bounds on the unknown rate $i$, which is then approximated by linear interpolation. A starting value is:

$i_{s}=\frac{(k / n)^{2}-1}{k}=\frac{(20 / 12)^{2}-1}{20}=0.0889=8.89 \%$, so 
$i_{1}=8 \%$ and $i_{2}=9 \%$ and corresponding factors are: $s_{12 \mid 0.08}=18.9771$ and $s_{12 \mid 0.09}=20.1407$. Substituting all these values to $(L I)$ for unknown interest rate $i$ we have:

$$
\begin{aligned}
& i=i_{1}+\left(i_{2}-i_{1}\right) \frac{s_{\left.12\right|_{i}}-s_{\left.12\right|_{1}}}{S_{\left.12\right|_{2}}-s_{\left.12\right|_{i}}}= \\
& =0.08+(0.09-0.08) \frac{20-18.9771}{20.1407-18.9771}= \\
& =0.0888=8.88 \% .
\end{aligned}
$$

We may check the accuracy of our answer by calculating the accumulated value of the deposits at $i=0.0888: S=250 s_{12 \mid 0.08}=$ $=4999.24$. Hence the unknown interest rate is $8.88 \%$.

\section{Example 2.}

You are offered a loan of $15000 €$ and agree to pay $1500 €$ annually for 15 years. What annual rate of interest does this loan charge?

We have $R=1500, A=15000, n=15, i=$ ? The way of solution will be similar to the previous example, but we use the formula $(P V)$. We obtain $1500 a_{15 \mid i}=15000 \Rightarrow a_{15 \mid i}=10=k$. The starting value is determined as: $i_{s}=\frac{1-(k / n)^{2}}{k}=\frac{1-(10 / 15)^{2}}{10}=$ $0.0556=5.56 \%$, interest rate values that differ by $1 \%$ are: $i_{1}=5 \%$ and $i_{2}=6 \%$ and corresponding two factors are: $a_{15 \mid 0.05}=10.3797$ and $a_{15 \mid 0.09}=9.7122$. Now using linear interpolation formula $(L I)$ for the unknown rate of interest we obtain:

$$
\begin{aligned}
& i=i_{1}+\left(i_{2}-i_{1}\right) \frac{a_{15 \mid i}-a_{\left.15\right|_{i}}}{a_{15||_{2}}-a_{\left.15\right|_{i}}}= \\
& =0.05+(0.06-0.05) \frac{10-10.3797}{9.7122-10.3797}= \\
& =0.05569=5.57 \% .
\end{aligned}
$$

\subsection{Bonds' Yield to Maturity}

In the section 2.3. we introduced three approximative methods for computation of yield to maturity of bonds. If a more accurate answer is desired, these methods should be followed by the method of linear interpolation. This method requires determining market prices of a bond for two interest rates, such that one price is smaller and the other is greater than the given quoted price. To do this we use the formula $(M P)$. Linear interpolation, i.e., formula $(L I)$ is then used to find the unknown $i$. If convenient, the interpolation can be on a purchase price rather than a market price. Methods introduced in section 2.3. can be used to determine a starting point for the linear interpolation.

The following example illustrates the calculating of yield to maturity $i$.

\section{Example 3.}

A company issued bond with maturity time of 14 years, with the face value $2000 €$ with coupon rate $9.5 \%$, compounded semiannually. The market price of the bond is $1930 €$. Find the yield to maturity of this bond.

We have: $F=2000 €, P=1930 €, r=0.095 / 2, C=F r=95 €$, $n=14 \cdot 2=28, i=$ ?

The starting value of semiannual interest rate is will be found using, e.g., the bond Salesman's method: $i_{s}=\frac{C+(F-P) / n}{(F+P) / 2}=$ $=\frac{95+(2000-1930) / 28}{(2000+1930) / 2}=0.0496$, so annual effective interest rate (yield to maturity) compounded semi-annually is: $i_{s}^{(2)}=$ $=2 \cdot 0.0496=0.0992=9.92 \%$. Now we determine two values $i_{1}$, $i_{2}: i_{1}=\frac{9}{2} \%=0.045, i_{2}=\frac{10}{2} \%=0.050$ and corresponding market prices one smaller and the other greater than the given market price $1930 €$ :

$$
\begin{aligned}
P\left(i_{1}\right) & =F r a_{n \mid i}+\frac{F}{(1+i)^{n}}=95 \frac{1-(1+0.045)^{-28}}{0.045}+ \\
& +\frac{2000}{(1+0.045)^{28}}=2078.71 € \\
P\left(i_{2}\right) & =95 \frac{1-(1+0.050)^{-28}}{0.050}+\frac{2000}{(1+0.050)^{28}}= \\
& =1925.51 € .
\end{aligned}
$$

Now we are in the position to use the linear interpolation formula $(L I)$ reformulated to case in question:

$$
\begin{aligned}
& i=i_{1}+\left(i_{2}-i_{1}\right) \frac{P-P\left(i_{1}\right)}{P\left(i_{2}\right)-P\left(i_{1}\right)}=0.045+ \\
& +(0.050-0.045) \frac{1930-2078.71}{1925.51-2078.71}=0.04985 .
\end{aligned}
$$

It is visible that unknown annual yield to maturity is $i^{(2)}=2 \cdot 0.04985=0.0997=9.97 \%$.

\section{Conclusion}

In this article we introduced some parts of mathematics of finance where calculating of the unknown interest rate is needed and this is non-trivial. In such cases the numerical method of linear interpolation can be used. In this paper also some interdisciplinary interconnections among mathematical analysis, numerical mathematics and financial mathematics can be seen. Hence regardless of some opinions, the world of mathematics and finance is close-knit. 


\section{References}

[1] HUTKA, V., PELLER, F.: Financial Mathematics in Excel (in Slovak), Iura Edition, Bratislava, 2007, ISBN 978-80-8078-143-9.

[2] KUDELCIK, J. et al: Multimedia Script of Laboratory Practices, 15th Conference of Slovak Physicists, 2006, Congress Centre Academia, Stara Lesna, Bratislava, Slovak Physical Society, 2007, ISBN 978-80-969124-4-5, pp. 121-122.

[3] KUDELCIKOVA, M.: Mathematics of Finance, EDIS Zilina, 2008, ISBN 978-80-8070-946-4.

[4] ZIMA, P., BROWN, R. L.: Mathematics of Finance, McGraw-Hill, New York, 1996, ISBN 0-07-008203-0. 